\title{
ON DECOMPOSITION OF CONTINUA INTO APOSYNDETIC CONTINUA $\left({ }^{1}\right)$
}

\author{
BY \\ LOUIS F. MCAULEY(2)
}

Introduction. This paper generalizes certain methods of decomposition of compact metric continua due to R. L. Moore $[3 ; 4]$ and G. T. Whyburn $[9 ; 10 ; 13]$. While their methods yield acyclic continuous curves, hyperspaces are obtained here which are aposyndetic [1] continua. The concept of a continuum being aposyndetic is a generalization of the concept of continuous curves and was introduced in 1941 by F. B. Jones. In compact metric continua, this idea is equivalent to Whyburn's notion of semi-locally-connectedness $[14 ; 2]$. A continuum $M$, i.e., a closed and connected point set, is said to be aposyndetic at a point $p$ with respect to a point $x$ provided that there exists a subcontinuum $N$ of $M$ and an open subset $O$ of $M$ such that $M-x \supset N \supset O \supset p$. If $M$ is aposyndetic at a point $p$ with respect to each point $x$ of $M-p$, then $M$ is said to be aposyndetic at $p$. It is said that $M$ is aposyndetic if $M$ is aposyndetic at each of its points.

In an early paper [10], Whyburn made use of connected cuttings of a compact metric continuum $M$ to obtain a decomposition of $M$ into an acyclic continuous curve. Later, he made use of nonseparated cuttings [13] to obtain a decomposition of a continuous curve into a nondegenerate acyclic continuous curve. Certain of these theorems concerning nonseparated cuttings are generalized in obtaining an aposyndetic decomposition.

Moore [3] has obtained decomposition theorems by use of certain sets $M(P)$ defined as follows: For each point $P$ of a compact continuum $M$, let $M(P)$ denote the set of all points $X$ of $M$ such that there do not exist uncountably many different points each separating $P$ from $X$ in $M$. He proved the following theorem: If $M$ is a compact metric continuum and $G$ is the collection of all point sets $M(P)$ for all points $P$ of $M$, then $G$ is an upper semi-continuous collection of disjoint continua filling up $M$ and $G$ is an acyclic continuous curve with respect to its elements as points.

The definition of the sets $M(P)$ due to Moore may be generalized in the following manner: Suppose that $M$ is a continuum and that $p$ is a point of $M$.

Presented to the Society, under the titles On the aposyndetic decomposition of continua and Generalized upper semi-continuous collections as applied to aposyndetic decompositions of continua on November 28, 1952, and November 27, 1953, respectively; received by the editors December 29, 1954.

(1) A thesis submitted to the faculty of the University of North Carolina in partial fulfillment of the requirements for the Ph.D. degree, June, 1954.

(2) The author wishes to express his appreciation and indebtedness to Professor F. B. Jones for having stimulated this research through his teaching. 
Let $M(p)$ denote the set of all points $x$ of $M$ such that there does not exist an uncountable nonseparated $\left(^{3}\right)$ collection $M(p, x)$ of subsets of $M$ such that each element of $M(p, x)$ separates $p$ from $x$ in $M$. In case $M$ is a compact metric continuum, it is proved that if $G$ is the collection of subcontinua $C$ of $M$ such that $C$ is a component of $M(p)$ for some point $p$ in $M$, then $G$ is an upper semi-continuous collection of disjoint continua filling up $M$; and furthermore, with respect to its elements as points, $G$ is a compact aposyndetic metric continuum. Other decomposition theorems are proved for more general spaces.

It is noted that this generalization of the sets $M(P)$ combines the notion of nonseparated cuttings due to Whyburn with the definition of $M(P)$ due to Moore. However, the collection of all point sets $M(p)$ for each point $p$ in $M$ may not be nonseparated and each $M(p)$ may fail to separate $M$. By certain restrictions on the elements of the nonseparated collection $M(p, x)$ in the definition of $M(p)$, a decomposition $H$ of a compact metric continuum $M$ is obtained such that $H$ is a continuous curve. If each element of $M(p, x)$ is a point, then the definition of $M(p)$ reduces to that given by Moore.

Throughout this paper, $M$ denotes a connected separable $T_{1}$ space.

1. Nonseparated separators. The word "separator" is used instead of "cutting" since some authors distinguish between "cut point" and "separating point" of a continuum. A separator of $M$ is a subset $N$ of $M$ such that $N$ separates $M$ into two separated point sets, i.e., $M-N=H+K$ and $\bar{H} \cdot K$ $=H \cdot \bar{K}=0$. Let the letter $K$ denote an uncountable collection of nonseparated separators of $M$. Now, consider the following fundamental properties of $K$. It should be noted that the elements of $K$ are not necessarily closed point sets.

K1. There exist elements $a, b$, and $c$ of $K$ such that $c$ separates $a$ from $b$ in $M\left({ }^{4}\right)$.

K2. There exist elements $p$ and $q$ in $K$ and an uncountable subcollection $K_{0}$ of $K$ such that each element of $K_{0}$ separates $p$ from $q$ in $M$ (Cf. [10, p. 88, Theorem 1]).

K3. For $a$ in $K$, there exists $b$ in $K$ and an uncountable subcollection $K_{0}$ of $K$ such that each element of $K_{0}$ separates $a$ from $b$ in $M$.

K4. If some element of $K$ separates $M$ into more than two separated point sets, then the collection of all such elements of $K$ is countable $\left({ }^{5}\right)$.

K5. Suppose that $T$ is a nonseparated collection of separators of $M$ such that for $h$ in $T, h=a+b$ where $a$ and $b$ are two elements of $K$. Then $T$ is countable.

(3) A collection $C$ of subsets of $M$ will be called nonseparated if and only if (1) the elements of $C$ are disjoint and (2) no element of $C$ separates in $M$ two points belonging to any other one element of $C$. Cf. $[13 ; 9]$.

( ${ }^{\prime}$ Cf. [9, p. 43 (Theorem 1.5)]. If space is metric, then Property K1 follows by Whyburn's Theorem 1.5. His proof makes use of a metric. This is not necessary since 1.5 follows from Property K1.

(5) Property K4 is a generalization of a theorem due to Whyburn $[13$, p. $449(\beta)]$. 
Property K1 may be established by an indirect argument. If $K$ fails to have Property $\mathrm{K} 1$, then for two elements $p$ and $q$ in $K$, there exist separations $M-p=A+B$ and $M-q=C+D$ where $B+p$ and $D+q$ are disjoint point sets, each containing an open set. Thus, the separability of $M$ is contradicted by the existence of uncountably many disjoint open sets.

Without separability, Property K1 does not necessarily follow.

To prove that $K$ has Property K2, it may first be shown that there exist two points $a$ and $b$ of $M$ (not necessarily elements of $K$ ) and an uncountable subcollection $K_{0}$ of $K$ such that each element of $K_{0}$ separates $a$ from $b$ in $M$. It follows $\left[13\right.$, p. 446] that $K_{0}$ possesses a natural order $\left({ }^{6}\right)$.

If $K_{0}$ fails to have Property K2, then there exists an uncountable sequence $\alpha=h_{1}, h_{2}, h_{3}, \cdots, h_{z}, \cdots$ of elements of $K_{0}$ such that either (1) for each pair of subscripts $x$ and $y$ where $x<y, h_{x}$ precedes $h_{y}$ in the order from $a$ to $b$ in $M\left(h_{x}<h_{y}\right)$ or (2) for each pair $(x, y)$ where $x<y, h_{x}>h_{y}$. In either case, it follows that $M$ contains uncountably many disjoint open sets. This is impossible since $M$ is separable. Hence, $K$ has Property K2.

It is not difficult to prove Property K3. Property K4 may be proved by using the following lemma which will be used again.

Lemma 1.0. If a subset $g$ of $M$ separates $M$ into three separated sets $S_{1}, S_{2}$, and $S_{3}$, then any subset $C$ of $S_{3}$ which separates $S_{1}+g+S_{2}$ in $M$ must separate $g$ in $M$.

Proof. If Lemma 1.0 is false, then there is a separation $M-C=N(A)$ $+N(B)$ of $M$ into two separated sets containing subsets $A$ and $B$ of $S_{1}$ and $S_{2}$, respectively. Suppose that $N(A) \supset g$. Consequently, $M$ is the sum of two separated point sets $\left[N(A)+C+N(B) \cdot S_{3}\right]+\left[N(B) \cdot S_{1}+N(B) \cdot S_{2}\right]$. This is a contradiction. Hence, Lemma 1.0 is true. Note that separability is not needed.

A proof of Property K5 is given to emphasize that these results are obtained without assuming that the elements of $K$ are closed. For $h=a+b$ in $K_{1}$ where $a, b \in K$, there exist separations $M-a=A+B$ and $M-b=C+D$ where $C \supset A+a$ and $B \supset b$. Let $E$ denote $B \cdot C$. Hence, $C=A+a+E$. If $E \neq 0$, then (1) $E+a+b$ contains an open subset of $M$ and (2) $E \cdot K^{*}=0$ since $K$ is a nonseparated collection ( $\left.{ }^{7}\right)$. If $E=0$, then either $b \cdot a \neq 0$ or $\bar{a} \cdot b \neq 0$. Now, if $b \cdot a$ contains a point $p$ there exists an open set $R \supset p$ such that $R \cdot D=0$. Since $R \cdot b \neq 0$, there exists an open set $Z$ containing a point of $b$ such that $Z \cdot(A+D)$ $=0$. Hence, $a+b$ contains an open subset of $M$. Since $M$ is separable, it follows that $K$ is countable.

(6) A set or collection of sets $N$ is said to be ordered or to possess an order provided that a definition of "preceding" (indicated $<$ ) can be defined for each pair of elements which is asymmetric and transitive. Suppose that for $x \in N, P_{x}$ denotes the logical sum of all elements of $N$ preceding $x$ and $F_{x}$ the sum of all those preceded by $x$ (or following $x$ ). Then the order in $N$ is said to be a natural order if and only if for each $x \in N, P_{x} \cdot F_{x}=P_{x} \cdot F_{x}=0$. Cf. $[13 ; 9]$.

${ }^{(7)}$ The symbol $K^{*}$ denotes the logical sum of the elements of $K$. 
Lemma 1.1. There exists an uncountable subcollection $K_{1}$ of $K$ such that (1) $K-K_{1}$ is countable and (2) if $g$ and $h$ are elements of $K_{1}$, then there exists an uncountable subcollection $M(g, h)$ of $K_{1}$ such that (i) each element of $M(g, h)$ separates $g$ from $h$ in $M$ uniquely and (ii) $M(g, h)$ possesses a natural order $\left.{ }^{8}\right)$.

Proof. Suppose that Lemma 1.1 is false. By Property K4, there exists an uncountable subcollection $T$ of $K$ such that $K-T$ is countable and each element of $T$ separates $M$ uniquely. Now, for two elements $p$ and $q$ in $T, p$ is said to be in relation $R$ to $q$ if and only if there is at most a countable number of elements of $T$ each of which separates $p$ from $q$ in $M$. It follows that $R$ is an equivalence relation.

It will be proved that there are at most a countable number of equivalence classes defined by $R$ each of which contains at least two elements of $T$. Suppose that this is not true. Then with each equivalence class which contains at least two elements, associate exactly one pair of its elements. Let $W$ denote the collection of all such pairs of elements of $T$. It follows that $W$ is an uncountable nonseparated collection of separators of $M$. This is contrary to Property K5. Hence, $W$ is countable. Let $K_{1}$ denote the collection of all elements $g$ of $T$ such that $g$ is the only element in the equivalence class to which $g$ belongs. Then $K_{1}$ satisfies the conclusion of Lemma 1.1 .

Although lemmas due to Zarankiewicz [15] and Whyburn [9, p. 44] are not true even in a regular separable semi-metric topological space $\left({ }^{9}\right)$, it is interesting to compare them with Lemma 1.1. As an illustration, consider the following example.

EXAMPLE 1.2. Let the space $S$ consist of all points $x$ of the number plane $E$ such that either (1) $x \in X$ where $X$ denotes the $x$-axis or (2) $x$ lies on a line $L$ perpendicular to $X$ such that the Cartesian distance from $L$ to the $y$-axis is a rational number. If $x$ and $y$ denote two points and $X \supset x$, then define $D(x, y)$ to be $d(x, y)+A$ where $d(x, y)$ is the ordinary Cartesian distance and $A$ is the measure (in radians) of an angle between a line $L_{1}$ containing $x+y$ and a line $L_{2}$ perpendicular to $X$ at $x$ such that $0 \leqq A \leqq \pi / 2$. If $D(x, y)$ is not defined above for $x$ and $y$ in $S$, then $D(x, y)=d(x, y)$. Define each spherical neighborhood to be an open set. Let $N$ denote the set of points $x$ of $X$ such that the Cartesian distance from $x$ to the origin is an irrational number. Thus, $N$ is an uncountable subset of the regular, connected, separable, and semi-metric topological space $S$. Each point of $N$ separates $S$ uniquely and $N$ possesses a natural order. However, no point of $N$ is a limit point of $N$.

(8) Cf. Zarankiewicz [15] and Whyburn [9, p. 44 (2.1)]. Also, cf. Lemmas 2.3-2.5 of this paper.

The proof given here of Lemma 1.1 was suggested by R. D. Anderson.

${ }^{(9)}$ If each of the letters $x$ and $y$ denotes a point of a topological space $S$ and there is associated with $(x, y)$ exactly one non-negative real number $d(x, y)$ called the distance from $x$ to $y$ such that (1) $d(x, y)=d(y, x),(2) d(x, y)=0$ if and only if $x=y$, and (3) each limit point of each subset $M$ of $S$ is a distance limit point of $M$ and conversely, then the space $S$ is said to be a semimetric topological space. 
Yet, if $g$ and $h$ denote two points of $N$ and if $K$ denotes the collection of all points $x$ in $N$ such that $g \leqq x \leqq h$ in $N$, then $K=K_{1}$ satisfies the conclusion of Lemma 1.1 where " $M$ " replaces " $S$."

2. Point sets $M(p)$ and $S(g, x)$. Nonseparated collections of separators are used to define the point sets $M(p)$ which are generalizations of $\mathrm{R}$. L. Moore's sets $M(P)$. In defining the point sets $S(g, x)$ which are subsets of the sets $M(p)$, collections $C$ of sets $M(p)$ are used whose properties include the property that $C^{*}$ is the sum of a finite number of continua.

Definition 2.1. Suppose that $\mathrm{Y}$ is a point set property $\left({ }^{10}\right)$. For $p$ in $M$, let $M(p)$ denote the set of all points $x$ of $M$ such that there does not exist an uncountable nonseparated collection $M(p, x, Y)$ of subsets of $M$ each having Property Y such that each element of $M(p, x, Y)$ separates $p$ from $x$ in $M$.

It is not difficult to prove that the point sets $M(p)$ have the following properties.

M1. If $p$ and $x$ denote points of $M$ and $M(p) \cdot M(x) \neq 0$, then $M(p)$ $=M(x)$.

M2. For $p$ in $M$, at most a countable number of the elements of $K$ (an uncountable nonseparated collection of separators of $M$ each having Property Y) either intersect or separate $M(p)$ in $M$.

M3. If $p$ and $x$ are two points in $M$ and $M(p) \neq M(x)$, then there exists an uncountable nonseparated collection $M(p, x, Y)$ of separators of $M$ such that each element of $M(p, x, Y)$ separates $M(p)$ from $M(x)$ in $M$.

M4. For $p$ in $M, M(p)$ is a closed point set.

Next, consider certain subsets of $M(p)$ defined as follows.

Definition 2.2. Suppose that $H$ denotes the collection of all points sets $M(p)$ for the various points $p$ in $M$. Then, for $x \in g \in H$, let $S(g, x)$ denote the set of all points $y_{\xi}$ of $g$ such that there does not exist a subcollection $C$ of $H$ such that (1) $C^{*}$ is the sum of a finite number of continua and (2) $C^{*}$ separates $x$ from $y$ in $M$.

The following properties of the point sets $S(g, x)$ may be established.

S1. For $g$ in $H$ and $x$ in $g, S(g, x)$ is a closed point set.

S2. If $x$ and $y$ denote points of $g$ in $H$ and $S(g, x) \cdot S(g, y) \neq 0$, then $S(g, x)=S(g, y)$.

Consider the following simple example which shows that a set $S(g, x)$ as defined in Definition 2.2 is not necessarily $g$.

EXAmple 2.21. Let the space $S$ be the number plane minus the set of points $x$ such that $1<x<3$. Next, let $M=\sum_{i=1}^{\infty} g_{i}$ where, for each $i>3, g_{i}$ is the set of points $(x, 1 / i)$ such that $0 \leqq x \leqq 4, g_{1}$ and $g_{2}$ are the closed intervals $[0,1]$ and $[3,4]$, respectively, of the $x$-axis, and $g_{3}$ is a unit interval perpendicular to the $x$-axis at the point $(2,0)$. If $p \in g_{1}$, then $M(p)=g_{1}+g_{2}$. For $p$ in $M-\left(g_{1}+g_{2}\right), M(p)=p$. The collection $C$ of all points $x$ in $g_{3}$ is a subcollection of the collection $H$ of all sets $M(p)$ for the various points $p$ in $M$ such

(10) Property Y may be that each element of $M(p, x, Y)$ is a point set. On the other hand, it may be that each element of $M(p, x, Y)$ is locally connected. 
that $C^{*}$ is a continuum and $C^{*}$ separates $g_{1}$ from $g_{2}$ in $M$. Thus, if $g=g_{1}+g_{2}$, $x \in g_{1}$, and $y \in g_{2}$, then $S(g, x)=g_{1}$ and $S(g, y)=g_{2}$. Define the elements of $H-g, g_{1}$, and $g_{2}$ to be "points." A subset $R$ of these "points" is an open set if and only if $R^{*}$ is an open set in $M$. Thus, this hyperspace is an aposyndetic metric continuum which is not a continuous curve.

Notation. As before, let $H$ denote the collection of all point sets $M(p)$ for the various points $p$ in $M$. If $a$ and $b$ denote two elements of $H$, then let $M(a, b)$ denote an uncountable nonseparated collection of separators of $M$ each of which separates $a$ from $b$ in $M$. Now, let $Q(a, b)$ denote an uncountable nonseparated collection of subsets of $M$ such that

(1) each element of $Q(a, b)$ separates $a$ from $b$ in $M$ uniquely,

(2) for each element $q$ of $Q(a, b)$ there exists an element $m$ of $M(a, b)$ such that the subcollection $H(m)$ of all elements of $H$ which intersect $m$ has the property that $H(m)^{*}=q$,

(3) $Q(a, b)$ possesses a natural order, and

(4) if $p$ and $q$ are two elements of $Q(a, b)$, then there exists an uncountable subcollection $Q_{1}$ of $Q(a, b)$ such that each element of $Q_{1}$ separates $p$ from $q$ in $M$.

Lemma 2.3. If $a$ and $b$ denote two elements of $H$, there exists a collection $Q(a, b)$.

Proof. By Properties M3 and K4 and Lemma 1.1, there exists an uncountable nonseparated collection $M(a, b)$ of separators of $M$ such that (1) each element of $M(a, b)$ separates $a$ from $b$ in $M$ uniquely, (2) $M(a, b)$ possesses a natural order, and (3) if $g$ and $h$ are elements of $M(a, b)$, then there exists an uncountable subcollection $M(g, h)$ of $M(a, b)$ such that each element of $M(g, h)$ separates $g$ from $h$ in $M$. For each $g$ in $M(a, b)$, let $N(g)$ denote the minimum subcollection of $H$ such that no element of $H-N(g)$ contains a point of $g$. It follows that $N(g)$ separates $a$ from $b$ in $M$.

By Property M2, no element of $N(\mathrm{~g})$ contains a point from each of uncountably many elements of $M(a, b)$. It will be proved that $N(g)$ intersects at most a countable number of the elements of $M(a, b)$. Suppose that there exists (1) an uncountable subcollection $N_{1}(g)$ of $N(g)$, (2) an uncountable subcollection $H(a, b)$ of $N(a, b)$, and (3) a one-to-one correspondence between the elements of $N_{1}(g)$ and $H(a, b)$ such that if an element $n$ of $N_{1}(g)$ corresponds to an element $c$ of $H(a, b)$, then $n \cdot c \neq 0$. Let $n_{1}$ denote an element of $N_{1}(g)$. Now, let $M_{1}(a, b)$ denote the maximum subcollection of $M(a, b)$ such that no element of $M_{1}(a, b)$ intersects $n_{1}$. By Property M2, $M(a, b)$ $-M_{1}(a, b)$ is countable. There exists an element $n_{2}$ in $N(g)$ such that $n_{2}$ intersects some element of $M_{1}(a, b)$. Of course, $n_{1} \neq n_{2}$. Let $M_{2}(a, b)$ denote the maximum subcollection of $M_{1}(a, b)$ such that no element of $M_{2}(a, b)$ intersects $n_{2}$. Now, $M_{1}(a, b)-M_{2}(a, b)$ is countable. Consequently, there exists (1) an uncountable collection of elements of $N(g),(2)$ a well-ordering of this collection, $n_{1}, n_{2}, n_{3}, \cdots, n_{2}, \cdots$, and (3) a corresponding se- 
quence $M_{1}(a, b), M_{2}(a, b), M_{3}(a, b), \cdots, M_{z}(a, b), \cdots$, of uncountable subcollections of $M(a, b)$ such that (i) no element of $M_{x}(a, b)$ intersects $n_{z}$ for $x<z$ and (ii) there exists an element $g_{z}$ of $\prod_{x<z} M_{x}(a, b)$ which intersects $n_{z}$. For each $z$, associate exactly one such element $g_{z}$ with $n_{z}$. Let $\left[g_{z}\right]$ denote the uncountable collection containing exactly one element corresponding to $n_{z}$ for each $z$. By Properties K2 and M2 as well as the definition of $\left[g_{\beta}\right]$, there exist two elements $g_{\alpha}$ and $g_{\beta}$ where $\alpha<\beta$ and an uncountable subcollection $K$ of $\left[g_{z}\right]$ such that for $g_{z}$ in $K, \beta<z$, and $g_{z}$ separates $g_{\alpha}+n_{\alpha}$ from $g_{\beta}+n_{\beta}$ in $M$. By definition of $N(g)$, both $n_{\alpha}$ and $n_{\beta}$ intersect $g$. Thus, $g_{z}$ of $M(a, b)$ separates $g$ of $M(a, b)$ in $M$. This is impossible since $M(a, b)$ is nonseparated. Hence, $N(g) *$ intersects at most a countable number of the elements of $M(a, b)$.

Consider two elements $g$ and $h$ in $M(a, b)$ and the corresponding subcollections $N(g)$ and $N(h)$ of $H$. It follows from the properties of $M(a, b)$ that there exists $x$ in $M(a, b)$ which separates $N(g)^{*}$ from $N(h)^{*}$ in $M$ uniquely. Consequently, the collection $Q(a, b)$ of all point sets $N(g)^{*}$ for each $g$ in $M(a, b)$ is a nonseparated collection of separators of $M$. Since (4) also follows from properties of $M(a, b)$, Lemma 2.3 is true.

Lemma 2.4. There exists an uncountable nonseparated collection $\widetilde{Q}(a, b)$ of separators of $M$ having not only properties (1), (3), and (4) of $Q(a, b)$ but also (5) $\widetilde{Q}(a, b)$ is saturated $\left.{ }^{11}\right)$, (6) each element of $\widetilde{Q}(a, b)$ is a closed point set, (7) for $q$ in $\widetilde{Q}(a, b)$, there exists a subcollection $H(q)$ in $H$ such that $H(q)^{*}=q$, and (8) no element of $\widetilde{Q}(a, b)$ separates in $M$ an element $h$ of $H\left({ }^{12}\right)$.

Proof. By Lemma 2.3, there exists a collection $Q(a, b)$. Such a collection exists which has neither a first element nor a last element in the order from $a$ to $b$ in $M$. Consider $Q(a, b)$ to have this property.

For $q$ in $Q(a, b)$, let $P(q)$ denote the point set consisting of $q$ together with all points $x$ in $M$ such that no element of $Q(a, b)$ separates $x$ from $q$ in $M$ (cf. $[13, \S 5])$. It follows that the collection $\widetilde{Q}(a, b)$ of all point sets $P(q)$ for the various elements in $Q(a, b)$ is a nonseparated collection of separators of $M$ which is saturated. By use of Lemma 1.0, it follows that each element of $\widetilde{Q}(a, b)$ separates $a$ from $b$ in $M$ uniquely.

It is not difficult to show that $\tilde{Q}(a, b)$ has all properties indicated in Lemma 2.4.

The following lemma is stated without proof $\left({ }^{13}\right)$.

Lemma 2.5. If $M$ is a hereditarily separable semi-metric topological space

(11) A nonseparated collection $C$ of subsets of $M$ will be said to be saturated provided that for $c \in C$ and $p \in M-c$, there exists at least one element $g$ of $C$ which separates $p$ from $c$ in $M$.

(12) Lemmas 2.4 and 2.5 contain parts of a theorem due to Whyburn [9, p. $45(2.2)]$ which are true in $M$ although parts of his argument do not generalize to give a proof in a separable topological space.

(13) A proof may be obtained from preceding results and a theorem in a note of the author's on naturally ordered sets in semi-metric spaces submitted to the Proc. Amer. Math. Soc. 
and a collection $Q(a, b)$ exists, then there is a subcollection $Q$ of $Q(a, b)$ which not only has the properties of $\widetilde{Q}(a, b)$ in Lemma 2.4 but also $(9) Q(a, b)-Q$ is countable and (10) no element $k$ of the naturally ordered collection $Q$ contains a point which is not a condensation point [9] both of $P(k)^{*}$ and $F(k)^{*}$ where $P(k)$ is the collection of all predecessors of $k$ in $Q$ and $F(k)$ is the collection of all successors of $k$ in $Q$.

Example 1.2 shows that Lemma 2.5 is not true without the requirement of hereditary separability.

3. Upper semi-continuous collections. The notion of upper semi-continuous collections is generalized so that it can be used in a topological space which may fail to satisfy the first axiom of countability. Although the space $M$ may not be a Hausdorff space, with respect to certain subsets of $M$ as "points," the resulting hyperspace may be Hausdorff provided that the collection of "points" is upper semi-continuous. It seems that the basic notion of upper semi-continuity does not involve the first axiom of countability but separation by "open sets" of " points."

Let $U$ denote a collection of point sets.

Definition 3.1. A subcollection $R$ of $U$ is said to be a region in $U$ if and only if $R^{*}$ is an open set in $M$.

Definition 3.2. An element $g$ of $U$ is said to be a limit element of a subcollection $A$ of $U$ provided that every region $R$ in $U$ which contains $g$ also contains an element of $(A+g)-g$.

Definition 3.3. A collection $U$ of point sets is said to be upper semi-continuous provided that if (1) $A$ is a subcollection of $U$ and (2) $g$ and $h$ are two limit elements of $A$, then there exists a subcollection $B$ of $A$ such that $g$ is a limit element of $B$ and $h$ is not a limit element of $B$.

Suppose that $U$ denotes an upper semi-continuous collection of disjoint closed point sets which fills up $M\left({ }^{14}\right)$. Then the following statements are true.

U1. A necessary and sufficient condition that a subcollection $A$ of $U$ be closed in $U$ is that $A^{*}$ be closed in $M$.

U2. If $A$ is a subcollection of $U$ and $A^{*}$ is connected in $M$, then $A$ is connected in $U$.

4. Upper semi-continuous decompositions of $M$ into an aposyndetic continuum. There are two essential types of decompositions given in this section. One is by the collection $H$ of all point sets $M(p)$ for the various points $p$ of $M$ while the other is by the collection $G$ of all point sets $S(g, x)$ for the various elements $g$ in $H$ and the various points $x$ of $g$. Throughout this paper, $H$ and $G$ denote the collections described above. By suitably defining Property Y in the definition of the sets $M(p)$, other decompositions of $M$ may be obtained. In fact, a "spectrum" of decompositions may be obtained. This will be indicated later.

Notation. The ordered pair $(R, U)$ denotes a hyperspace whose " points"

${ }^{(14)}$ A collection $C$ of sets is said to fill up $M$ if and only if $C^{*}=M$. Cf. [3]. 
are the elements of an upper semi-continuous collection $U$ and whose "regions" are those in the collection $R$ of all regions in $U$ defined by Definition 3.1. If a special subcollection $V$ of $R$ is used to define a topology for a hyperspace whose "points" are the elements of $U$, then $(V, U)$ denotes this space.

THEOREM 4.1. The collection $H$ is an upper semi-continuous collection of disjoint closed point sets filling up $M$; and furthermore, with respect to the elements of $H$ as points, $(R, H)$ is a connected, aposyndetic, and separable Hausdorff space.

Proof. By Properties M3 and M4 as well as the definition of $H$, it follows that $H$ is a collection of disjoint closed point sets which fills up $M$.

Next, it will be proved that for two elements $g$ and $h$ in $H$, there exist disjoint regions $R(g)$ and $R(h)$ in $H$ containing $g$ and $h$, respectively. Consequently, $H$ is upper semi-continuous. By Lemma 2.4, there exists a collection $\widetilde{Q}(g, h)$ having the properties indicated there. Thus, if $e$ is an element of $\widetilde{Q}(g, h)$, then there exists a unique separation $M-e=S(g)+S(h)$.

Now, there exists a subcollection $H(e)$ of $H$ such that $H(e)^{*}=e$. Furthermore, $e$ fails to separate an element of $H$ in $M$. Consequently, the collections $R(g)$ and $R(h)$ of all elements in $H$ which intersect $S(g)$ and $S(h)$, respectively, have the property that $R(g)^{*}=S(g)$ and $R(h)^{*}=S(h)$. Since $e$ is a closed point set, it follows by Definition 3.1 that $R(g)$ and $R(h)$ are regions in $H$ containing $g$ and $h$, respectively. It follows easily that $(R, H)$ is a Hausdorff space.

By Property U2, the special region $R(g)$ in $H$ described above is connected in $H$. Similarly, $(R, H)$ is connected. Also, the closure $\bar{R}(g)$ of $R(g)$ in $H$ is a continuum which fails to contain $h$. Hence, $(R, H)$ is aposyndetic at $g$ with respect to $h$. Since $g$ and $h$ denote two elements of $H$, it follows that $(R, H)$ is aposyndetic. The separability of $M$ implies the separability of $(R, H)$.

REMARK. Observe that if one considers a special class $V_{1}$ of regions in $H$ and then defines a topology for $H$ with respect to these regions, then one obtains that $\left(V_{1}, H\right)$ has all the properties prescribed by Theorem 2.1 as well as being regular. Consider the following Theorem 4.2.

NotAtion. Let $V$ denote the collection of all regions $R$ in $H$ such that there exist $a$ and $b$ in $H$, a collection $\widetilde{Q}(a, b)$ having properties indicated in Lemma 2.4, an element $e$ in $\Phi(a, b)$, and a separation $M-e=S(a)+S(b)$ such that the collection $R(a)$ of all elements of $H$ which intersect $S(a)$ has the property that $R(a)=R$. Now, let $V_{1}$ denote the collection of all regions $R$ such that either (1) $R$ is in $V$ or (2) $R$ is the collection of all elements common to a finite number of regions belonging to $V$.

THEOREM 4.2. The hyperspace $\left(V_{1}, H\right)$ is an aposyndetic, connected, separable, and regular Hausdorff space.

Proof. It suffices to give only a proof of the regularity of $\left(V_{1}, H\right)$. If $R$ is an element of $V_{1}$ containing $a$ in $H$, then there exists a finite number 
of subcollections $\widetilde{Q}_{i}\left(a_{i}, b_{i}\right), 1 \leqq i \leqq n$, each having the properties of $\tilde{Q}(a, b)$ referred to above such that for $1 \leqq i \leqq n,(1) R \supset a_{i},(2) H-\bar{R} \supset b_{i},(3) \tilde{Q}_{i}\left(a_{i}, b_{i}\right)$ $\supset e_{i}$ such that $M-e_{i}=S\left(a_{i}\right)+S\left(b_{i}\right)$, and (4) $R=\prod_{i=1}^{n} R\left(a_{i}\right)$ where $R\left(a_{i}\right)$ denotes the collection of all elements of $H$ which intersect $S\left(a_{i}\right)$. Since $\tilde{Q}_{i}\left(a_{i}, b_{i}\right)$ is saturated and each element of $\tilde{Q}_{i}\left(a_{i}, b_{i}\right)$ separates $M$ uniquely for each $i$, there exists $h_{i}$ in $\widetilde{Q}_{i}\left(a_{i}, b_{i}\right)$ such that (1) $M-h_{i}=T_{i}(a)+T\left(e_{i}\right)$ and (2) $T\left(e_{i}\right) \supset S\left(b_{i}\right)$. It follows that the collection $R_{i}(a)$ of all elements of $H$ which intersect $T_{i}(a)$ is a region in $H$ belonging to $V_{1}$. By the properties of $\widetilde{Q}_{i}\left(a_{i}, b_{i}\right)$, there exists a subcollection $H\left(h_{i}\right)$ of $H$ such that $H\left(h_{i}\right)^{*}=h_{i}$ which is closed in $M$. Also, $R\left(h_{i}\right)$ is closed in $H$. Since $S\left(a_{i}\right) \supset a, T_{i}\left(e_{i}\right) \supset S\left(b_{i}\right)$, and $e+S\left(a_{i}\right)$ is connected, it follows that $S\left(a_{i}\right) \supset h_{i}$. Hence, $R\left(a_{i}\right) \supset H\left(h_{i}\right)$. Now, the boundary of $R_{i}(a)$ is a subcollection of $H\left(h_{i}\right)$. Thus, $\prod_{i=1}^{n} R_{i}(a)=R_{1}$ is a region in $H$ containing $a$ such that $R \supset \bar{R}_{1}$. Since $R_{1}$ is in $V_{1},\left(V_{1}, H\right)$ is regular.

Theorem 4.3. The collection $G$ [of all sets $S(g, x)$ for the various elements $g$ in $H$ and the various points $x$ in $g$ ] is an upper semi-continuous collection of disjoint closed point sets filling up $M$; and furthermore, with respect to the elements of $G$ as points, $(R, G)$ is a connected, aposyndetic, and separable Hausdorff space.

Proof. Let $a$ and $b$ denote two elements of $G$. Now, there exist $g$ and $h$ in $H$ such that $g \supset a$ and $h \supset b$.

If $g \neq h$, then by arguments analogous to those given in the proof of Theorem 4.1, there exist disjoint regions $R(a)$ and $R(b)$ in $G$ containing $a$ and $b$, respectively, having properties desired to establish this theorem.

If $g=h$, then by Definition 2.2 there exists a subcollection $C$ of $H$ such that (1) $C^{*}$ is the sum of a finite number of continua and (2) $C^{*}$ separates $a$ from $b$ in $M$. By Property U1, $C$ is closed in $H$. Now, there exists a separation $M-C^{*}=S(a)+S(b)$ such that no element of $G$ intersects both $S(a)$ and $S(b)$. Let $R(a)$ denote the collection of all elements in $G$ which intersect $S(a)$. Similarly, define $R(b)$. Thus, $R(a)$ and $R(b)$ are regions in $G$ containing $a$ and $b$, respectively, such that $R(a)^{*}=S(a)$ and $R(b)^{*}=S(b)$. It follows that $G$ is an upper semi-continuous collection of disjoint closed point sets filling up $M$; furthermore, $(R, G)$ is a Hausdorff space.

It remains to be proved that $(R, G)$ is aposyndetic when $g=h$. Now, as above, there exists a subcollection $C$ of $H$ such that (1) $C^{*}=\sum_{i=1}^{n} N_{i}$ where $n$ is a positive integer and $N_{i}$ is a subcontinuum of $M$ for $1 \leqq i \leqq n$ and (2) there exists a separation $M-C^{*}=S(a)+S(b)$. Now, it follows that there exists a subcollection $B$ of $G$ such that $B^{*}=C^{*}$. Defining $R(a)$ and $R(b)$ as above, suppose that $R(a)+B$ is not connected in $G$. Then $R(a)+B$ is the sum of two separated subcollections $H_{11}$ and $H_{12}$ of $G$. Either $H_{11} \supset a$ or $H_{12} \supset a$. Neither $H_{11}^{*}$ nor $H_{12}^{*}$ fails to contain a point of $B^{*}$. For if $H_{1 i}^{*} \cdot B=0$, $i=1$ or 2 , then $M=H_{1 i}^{*}+\left[B^{*}+H_{1 j}^{*}+S(b)\right], i+j=3$, which is the sum of two separated point sets. This is contrary to the hypothesis that $M$ is connected. It follows that the collection $G\left(N_{i}\right)$ of all elements $x$ of $G$ which intersect $N_{i}$ 
is connected for $1 \leqq i \leqq n$. Now, if $N_{i} \cdot H_{1 j}^{*} \neq 0$ for some $i$ and $j=1$ or 2 , then $H_{1 j} \supset G\left(N_{i}\right)$. Consequently, for $j=1$ or $2, H_{i j}$ contains at most $n-1$ of the subcollections $G\left(N_{i}\right)$. Thus, there exists a separation $R(a)+B=B_{1}+B_{2}$ such that (1) $B_{1} \supset a$, (2) $B_{1}$ contains $m \geqq 1$ of the collections $G\left(N_{i}\right)$ for $1 \leqq i \leqq n$, and (3) there exists no separation $R(a)+B=H_{1}+H_{2}$ where $H_{1} \supset a$ and $H_{1}$ contains less than $m$ of the collections $G\left(N_{i}\right)$. If $B_{1}$ is not connected in $G$, then $B_{1}=B_{11}+B_{12}$ where $B_{11} \supset a$. By an argument similar to one given above, it follows that $B_{11}$ contains at most $m-1$ of the collections $G\left(N_{i}\right)$. This is impossible. Hence, $B_{1}$ is connected in $G$. By Property U1, $B$ is closed in $G$. It follows that $B-B_{1} \cdot B$ is a region in $G$. Consequently, $\bar{B}_{1}$ is a continuum in $G-b$ which contains a region in $G$ containing $a$. Hence, $(R, G)$ is aposyndetic at $a$ with respect to $b$. It follows that Theorem 4.3 is true.

REMARK. An upper semi-continuous decomposition of either $(R, H)$ or $(R, G)$ by collections consisting of sets defined for $(R, H)$ and $(R, G)$ like the sets $M(p)$ and $S(g, x)$, respectively, yields the same hyperspace. This is stated in the following theorem.

Notation.' The letters $H$ and $G$ denote collections defined as above. Let $H H[H G]$ denote the collection of all point sets $H(p)[G(p)]$ for the various points $p$ in $(R, H)[p$ in $(R, G)]$; and $G H[G G]$ denote the collection of all point sets $S(g, x)$ for the various elements $g$ in $H H[g$ in $H G]$ and the various points $x$ in $g$.

TheORem 4.4. If Property $\mathrm{Y}$ is the property of being a point set $\left({ }^{15}\right)$, then $H H=H$ and $G H=G$.

Proof. First, consider $H H$. If $a, b \in H, a \neq b$, then by Lemma 2.4 there exexists a collection $Q(a, b)$. From the properties of $Q(a, b)$ and Definition 2.1, it follows that $H(a)^{*}=a$. Thus, $H H=H$.

Consider $G H$. Since $H H=H$, the definition of $G H$ is the definition of $G$. Consequently, $G H=G$.

In the following theorem, a decomposition of $M$ into an aposyndetic continuum is given which leaves aposyndetic continua invariant.

Theorem 4.5. Suppose that B denotes the collection of all elements in $G$ which contain points $x$ and $y$ such that $M$ is not aposyndetic at $x$ with respect to $y$; furthermore, suppose that $A$ denotes the collection of all point sets $k$ such that either (1) $k \in \bar{B}$ (the closure of $B$ in $G$ ) or (2) $k \in M-(\bar{B})^{*}$. Then the collection $A$ is an upper semi-continuous collection of disjoint closed point sets filling up $M$; and furthermore, $(R, A)$ is a connected, aposyndetic, and separable Hausdorff space.

A proof of Theorem 4.5 may be obtained by arguments analogous to those already given.

(15) In case $M$ is perfectly separable, the restriction on Property $\mathrm{Y}$ may be omitted and Lemma 2.5 may be used to establish the resulting theorem. 
5. A necessary and sufficient condition for an aposyndetic metric hyperspace when $M$ is perfectly separable. Many theorems which have restrictions on the elements of an upper semi-continuous decomposition yield desirable hyperspaces. Unfortunately, these restrictions are not guaranteed even though space is perfectly separable. However, consider the following theorem and example.

THEOREM 5.1. Suppose that $M$ is perfectly separable. Then a necessary and sufficient condition that the space $\left(V_{1}, H\right)$ [See Theorem 4.2] be a connected aposyndetic perfectly separable metric space is that $\left(V_{1}, H\right)$ satisfy the first axiom of countability.

Proof. Since a metric space satisfies the first axiom of countability, it suffices to prove only the necessity.

By Theorem 4.2, $\left(V_{1}, H\right)$ is an aposyndetic regular Hausdorff space. In this proof that $\left(V_{1}, H\right)$ is perfectly separable and consequently metric, $\left(V_{1}, H\right)$ is embedded in a space which may contain "contiguous points." For each $g$ in $H$, associate exactly one sequence $\left\{R_{i}(g)\right\}$ of regions in $V_{1}$ closing down on $g$. Let $\left\{D_{i}\right\}$ denote a sequence of open sets in $\left(V_{1}, H\right)$ such that for each $i, D_{i} \supset \bar{D}_{i+1}$ and $\amalg D_{i}=0$. Furthermore, let $P$ denote the collection of all such monotonic descending sequences $\left\{S_{i}\right\}$ of open sets in $\left(V_{1}, H\right)$ such that (1) for each $i$, there exists $m(i)$ such that $D_{m(i)} \supset S_{i}$ and (2) for each $i$, there exists $n(i)$ such that $D_{i} \supset S_{n(i)}$. Define $P$ to be a point; and furthermore, associate with each such point $P$ exactly one sequence $\left\{D_{i}\right\}$ called the defining sequence of $P$.

Now, consider the following definition of neighborhoods for these newly defined points. For each $i$, a point $P$, and a defining sequence $\left\{D_{i}\right\}$ of $P$, a neighborhood $N_{i}(P)$ of $P$ is defined to be the point set consisting of all elements of $H$ which lie in $D_{i}$ and all points $Q$ such that for some positive integer $k, D_{i} \supset S_{k}$ where $\left\{S_{i}\right\}$ is the defining sequence of $Q$. If $g \in H$, then for each $i$, a neighborhood $N_{i}(g)$ consists of all elements of $H \cdot R_{i}(g)$ and all points $Q$ such that for each $j, R_{i}(g) \cdot S_{j} \neq 0$, where $\left\{S_{i}\right\}$ defines $Q$. Let $(D, H)$ denote the space whose points are the elements of $H$ and all points $P$ with neighborhoods defined above. Limit points are defined in the usual way.

A distance function will be defined for certain points of $(D, H)$ in the following manner. Let $N$ denote $(D, H)-H$ and $\left\{C_{i}\right\}$ denote a countable basis for the original space $M$. Suppose that $g \in H, P \in N$, and that there exist integers $i$ and $j$ such that $N_{i}(g) \cdot N=0$. Then let $C_{n(i)}$ denote the open set in $\left\{C_{i}\right\}$ with smallest subscript such that (1) $R_{i}(g) \supset C_{n(i)}$ and (2) $C_{n(i)}$ intersects both $M-g$ and $g$; furthermore, let $C_{n(j)}^{\prime}$ denote the open set of $\left\{C_{i}\right\}$ of least subscript such that $D_{j} \supset C_{n(j)}$ where $R_{i}(g)$ and $D_{j}$ belong to the sequences $\left\{R_{i}(g)\right\}$ and $\left\{D_{i}\right\}$ associated with $g$ and $P$, respectively. Let $M=\min [n(i), n(j)]$ for all pairs of disjoint neighborhoods $N_{i}(g)$ and $N_{j}(P)$. Define the distance $D(g, P)$ to be $1 / m$. If $N_{i}(g) \cdot N_{j}(P) \neq 0$ for each $i$ and $j$, then define $D(g, P)$ to be 0 . For $g, h \in H$, define $D(g, h)$ in a similar way. 
With respect to the distance function $D$ defined above, certain limit points are invariant. If $P \in N$ is a neighborhood limit point of a subcollection $C$ of $H$, then $P$ is a distance limit point of $C$ and conversely. Furthermore, a point $g$ in $H$ which is a distance limit point of a subset $Q$ of $N$ is a neighborhood limit point of $Q$. If $g \in H$ is a neighborhood limit point of a subset $C$ of $H$, then $g$ is a distance limit point of $C$ and conversely.

It will now be shown by use of this distance function that $(D, H)$ possesses a countable basis. For $x \in(D, H)$ and each $i$, let $U_{1 / i}(x)$ be a spherical neighborhood of $x$ while $u_{1 / i}(x)=U_{1 / i}(x) \cdot H$ is a spherical neighborhood of $x$ in $\left(V_{1}, H\right)$. If each of $m$ and $n$ is a positive integer, then let $H_{m n}$ be the set, if it exists, of all points $g$ in $\left(V_{1}, H\right)$ such that there exists $i$ satisfying (A) $U_{1 / m}(g)$ $\supset N_{i}(g)$ and (B) $U_{1 / m}(g) \supset \bar{R}_{i}(g) \supset R_{i}(g) \supset u_{1 / n}(g)$. Since $H$ is hereditarily separable, there exists a countable dense subset $G_{m n}$ of $H_{m n}$. Thus, let $R_{m n}$ denote a countable collection of regions in $V_{1}$ such that for $g$ in $G_{m n}$, there exists $i$ and $R_{i}(g)$ in $R_{m n}$ satisfying (A) and (B) above. For $g$ in $H, m$ and $n$ exist such that $g \in H_{m n}$. Hence, $\left[R_{m n}\right]$ covers $H$. Now, let $T$ be a countable subcollection of open subsets of $\left(V_{1}, H\right)$ such that (1) if $R \in R_{m n}$ for some $m$ and $n$, then $R \in T$ and (2) the intersection of two elements of $T$ is an element of $T$. It will be shown that $T$ is a basis for $H$. Suppose that there exists $k$ and $R_{k}(g) \supset g$ in $H$ such that there does not exist $R \in T$ such that $R_{k}(g) \supset R$ $\supset g$. For some $m_{1}$, there exists $g_{1} \in G_{1 m_{1}}$ and $R_{m_{1}}\left(g_{1}\right) \in R_{1 m_{1}}$ such that $R_{m_{1}}\left(g_{1}\right) \supset g$. Thus, $R_{m_{1}}\left(g_{1}\right) \cdot\left[H-R_{k}(g)\right] \neq 0$. It follows for each $i$, that there exists $m_{i}$, $g_{i} \in G_{i m_{i}}$, and a region $R_{m_{i}}\left(g_{i}\right) \in R_{i m_{i}}$ which contains $g$ such that for each positive integer $n, \prod_{i=1}^{n} R_{m_{i}}\left(g_{i}\right) \cdot\left[H-R_{k}(g)\right] \neq 0$. Let $Q_{n}=\prod_{i=1}^{n}\left\{R_{m_{i}}\left(g_{i}\right)\right.$ - $\left.\left[H-\bar{R}_{k+1}(g)\right]\right\}$ for each $n$. If $\pi \bar{Q}_{n}$ contains a point $x \in\left[H-R_{k+1}(g)\right]$, then $\bar{R}_{m_{i}}\left(g_{i}\right) \supset x$ for each $i$. Thus, $\left\{D\left(g_{i}, x\right)\right\} \rightarrow 0$ and $\left\{g_{i}\right\} \rightarrow x$. Also, $\left\{D\left(g_{i}, g\right)\right\} \rightarrow 0$ and $\left\{g_{i}\right\} \rightarrow g$. This is impossible. Hence, $\Pi \bar{Q}_{n}=0$. For each positive integer $n$, there exists an open set $Q_{1 n}$ in $\left(V_{1}, H\right)$ such that $Q_{1 n} \cdot Q_{n} \neq 0, \prod_{i=1}^{n} R_{m_{i}}\left(g_{i}\right)$ $\supset Q_{1 n}$, and $Q_{n} \supset \bar{Q}_{1 n}$. It follows that for each $n$ and each $i$ where $1<i \leqq n$, there exists an open set $Q_{i n}$ in $\left(V_{1}, H\right)$ such that $Q_{i n} \cdot Q_{n} \neq 0$ and $Q_{(i-1)} \supset \bar{Q}_{i n}$ where $Q_{0 n}=Q_{n}$. Now, for each $i$, let $S_{i}=\sum_{n=i}^{\infty} Q_{i n}$. Thus, $\left\{S_{i}\right\}$ is an element of a collection $P$ which is a point of $N$. Since $P \in N_{i}\left(g_{i}\right)$ and $D\left(g_{i}, P\right)<1 / i$ for each $i,\left\{g_{i}\right\} \rightarrow P$. It also follows that $N_{k+1}(g) \cdot N_{i}(P)=0$ for each $i$. Consequently, $\left\{g_{i}\right\}$ cannot converge to both $P$ and $g$. Hence, $T$ is a countable basis for $\left(V_{1}, H\right)$.

Since $\left(V_{1}, H\right)$ is a regular perfectly separable topological space, it follows by theorems due to Urysohn [8] and Tychonoff [7] that $\left(V_{1}, H\right)$ is metric.

REMARK. Theorem 5.1 holds for either a regular space $(R, H)$ or $(R, G)$. Even though $M$ satisfies the hypothesis of Theorem 5.1, i.e., $M$ is a perfectly separable metric space, it is not necessarily true that $\left(V_{1}, H\right)$ satisfies the first axiom of countability. This is shown in the following example.

EXAMPLE 5.2. In the number plane $E$, let $M=\sum g_{i}$ where $g_{1}$ denotes the set of points $(x, 0)$ where $0 \leqq x \leqq 1 ; g_{2}$ is all $(0, y)$ such that $-1<y<1 ; g_{3}$ is all $(x, y)$ where $-1 \leqq x<0$ and $y=\sin (1 / x) ; g_{4}$ is all $(x, 1)$ such that $0<x \leqq 1$; 
and for each integer $i>4, g_{i}$ is all $(1 / i, y)$ where $0<y<1$. An open set in $M$ is the intersection of an open set in $E$ with $M$. It follows that $M$ is a locally compact metric continuum which is perfectly separable. Now, $g_{2}$ is the only element of $H$ which is not a point. It will be shown that $\left(V_{1}, H\right)$ does not satisfy the first axiom of countability. Suppose that there exists a sequence $\left\{R_{i}\right\}$ of regions in $V_{1}$ which defines the topology of $\left(V_{1}, H\right)$ at $g_{2}$. There exists a sequence $\left\{p_{i}\right\}$ of points and a positive integer $k$ such that (1) for each $i>k$, $p_{i} \in g_{i} \cdot R_{i}$ and (2) $\sum p_{i}$ has no limit point in $M$. Thus, $\left\{p_{i}\right\} \rightarrow(0,1)$ in $E$. From the definition of $V_{1}$ as well as Definition 2.1, it follows that there exists a region $R$ in $V_{1}$ containing $g_{2}$ such that $R \cdot \sum p_{i}=0$. Hence, there exists no $i$ such that $R \supset R_{i}$. Thus, $\left(V_{1}, H\right)$ fails to satisfy the first axiom of countability.

REMARK. Except in a compact metric continuum $M$, the first axiom of countability as well as local compactness may fail to be properties which hold for any of the hyperspaces $\left(V_{1}, H\right),(R, H)$, and $(R, G)$. Note that the following theorem requires that the first axiom of countability be satisfied by the hyperspace; otherwise, the conclusion is not true.

TheOREM 5.3. Suppose that $M$ is a locally compact metric continuum and that $(R, G)$ satisfies the first axiom of countability. Then $(R, G)$ is a locally compact aposyndetic metric continuum.

Proof. Since $M$ is locally compact, $M$ is both perfectly separable and hereditarily separable. By Theorem $4.3,(R, G)$ is a connected aposyndetic Hausdorff space.

It will be shown that $(R, G)$ is regular. For each $g$ in $G$, there exists a monotonic descending sequence of regions $\left\{R_{i}(g)\right\}$ in $G$ each containing $g$ such that for a region $R \supset g$, there exists $n$ such that $R \supset R_{n}(g)$. Suppose that there exists a region $R \supset g$ and no region $R_{1}$ such that $R \supset \bar{R}_{1} \supset g$. Consider a collection $\left[Q_{i}\right]$ of open sets in $M$ which covers $g$ such that for each $i, R^{*} \supset \bar{Q}_{i}$ and $\bar{Q}_{i}$ is compact. Also, there exists $n$ such that $R \supset R_{i}(g)$ for each $i \geqq n$. For convenience, suppose that $n=1$. Since $R \supset \bar{R}_{i}(g)$ for no $i$, there exists a sequence $\left\{g_{i j}\right\}$ of elements of $R_{i}(g)$ which converges to $g_{i}$ in $\bar{R}_{i}(g)-R \cdot \bar{R}_{i}(g)$. Now, for each $i$, there exists $n_{i}$ such that $g_{i n_{i}} \cdot\left(\sum_{k=1}^{i} \bar{Q}_{k}\right)=0$ since $G$ is upper semi-continuous and $\bar{Q}_{k}$ is compact. Thus, $\left\{g_{i n_{i}}\right\} \rightarrow g$. It follows that $\left(\sum g_{i n_{i}}\right)$ has a limit point $x$ in $g$; furthermore, there exists $k$ such that $Q_{k} \supset x$. For infinitely many integers $i, Q_{k} \cdot g_{i n_{i}} \neq 0$. This is a contradiction. Therefore, $(R, G)$ is regular.

In a similar way, it may be shown that $(R, G)$ is locally compact.

By an argument analogous to the one given for Theorem 5.1, it may be shown that $(R, G)$ is metric.

6. Decompositions of compact metric continua into aposyndetic continua. Since much is known about compact metric continua $M$, decompositions of $M$ seem more interesting. It is in such a space that one can find a variety of applications of this theory. Also, it is easy to see how this work ties in with results of Moore and Whyburn. Here the elements of $G$ are subcontinua of 
$M$, in fact, each element of $G$ is a component of some element of $H$. Certain definitions of Property Y yield $H=G$ while others yield hyperspaces $(R, G)$ which are locally connected.

First, consider the following example of a compact metric continuum $M$ in the number plane such that $M$ contains a point $p$ where $M(p)$ is not connected.

EXAmple 6.1. Suppose that (1) $J$ denotes a circle in the number plane whose center is a point $p$, (2) $x$ and $y$ are two points of $J$, and (3) $A$ and $B$ are two open arcs such that $A+p+B$ is an open arc lying in the interior $I(J)$ of $J$ whose end points are $x$ and $y$. Now, $I(J)$ is separated by $A+p+B$ into two connected domains $I_{1}$ and $I_{2}$. Let $C, D$, and $E$ be disjoint open arcs in $I(J)$ with end points $p$ and $x$ such that (1) $B \cdot C=a_{2}, B \cdot D=a_{3}$, and $B \cdot E$ $=a_{1}+a_{4}$ where $p<a_{1}<a_{2}<a_{3}<a_{4}<y$ on the $\operatorname{arc} p B y$ in the order from $p$ to $y$, (2) each of $C \cdot I_{i}$ and $D \cdot I_{i}$ is one open arc for $i=1$ and $i=2$, (3) $A \cdot E$ is a point, and (4) each of $E \cdot I_{1}$ and $E \cdot I_{2}$ is the sum of two open arcs having no common end point. Let $J_{1}$ and $J_{2}$ denote simple closed curves lying in $N=J+A+B+C+D+E+p$ such that (1) $B \cdot J_{1} \cdot J_{2}$ contains an open arc $S$ with end points $a$ and $b$, (2) $N \cdot\left[I\left(J_{1}\right)+I\left(J_{2}\right)\right]=0$, and (3) $I\left(J_{1}\right) \cdot I\left(J_{2}\right)=0$. Thus, the boundary of $I\left(J_{1}\right)+S+I\left(J_{2}\right)$ is a simple closed curve $J_{3}$. Also, let (1) $\left\{x_{i}\right\}$ and $\left\{y_{i}\right\}$ denote sequences of points of $S$ converging to $a$ and $b$, respectively, such that $a<x_{i+1}<x_{i}<y_{i}<y_{i+1}<b$ on $a S b$ for each $i$ and $j$, and (2) $\left\{C_{i}\right\}$ denote a sequence of disjoint simple closed curves lying in $I\left(J_{3}\right)$ such that (a) for each $i, C_{i} \cdot S=x_{i}+y_{i}$ while each of $C_{i} \cdot I\left(J_{1}\right)$ and $C_{i} \cdot I\left(J_{2}\right)$ is an open arc, and (b) the limiting set of $\left\{C_{i}\right\}$ is $J_{3}$, in fact, $J_{3}+S+\sum C_{i}$ is an aposyndetic continuum. Finally, let $M$ denote a space whose points are those of the arcs and simple closed curves described above including all possible choices for $J_{1}$ and $J_{2}$. It follows that $M$ is a compact aposyndetic continuum in the number plane. Furthermore, $M(p)=p+x$.

REMARK. By use of Example 6.1, it may be shown that there exists a compact continuum $M$ in the plane containing a point $x$ such that $M(x)$. is an infinite totally disconnected point set.

The following theorem shows that the sets $S(g, x)$ are components of the sets $M(p)$.

THEOREM 6.2. If $x \in g \in H$, then $S(g, x)$ is the component of $g$ which contains $x$.

Proof. Let $C$ denote the component of $g$ which contains $x$. By Definition 2.2, $S(g, x) \supset C$. It will be shown that $C \supset S(g, x)$. Suppose that $C_{1}$ is a component of $g-C$. Since $C$ and $C_{1}$ are disjoint closed subsets of $M$, there exists a closed and compact point set $B$ which separates $C$ from $C_{1}$ in $M$. Let $H_{B}$ denote the collection of all elements of $H$ which intersect $B$. Thus, $H_{B}^{*}$ separates $C$ and $C_{1}$ in $M$. For $h$ in $H_{B}$, there exists an uncountable nonseparated collection $M(g, h, Y)$ of separators of $M$ each of which separates $g$ from $h$ in $M$ by Property M3 and the definition of $H$. Either by use of Lemma 2.5 or 
by a theorem due to Whyburn [9, p. $45(2.2)]$, there exists a collection $Q(g, h)$ having properties described in Lemma 2.5. Thus, there exists $q \in Q(g, h)$ such that (1) there exists a subcollection $H(q)$ of $H$ such that $H(q)^{*}=q$ and (2) $H(q)$ separates $(R, H)$ into two disjoint connected open sets $R(g)$ and $R(h)$ containing $g$ and $h$, respectively; furthermore, $R(g)^{*}$ and $R(h)^{*}$ are disjoint open subsets of $M$. From the definition of $Q(g, h)$, it follows that $q+R(h)^{*}$ is a continuum in $M-g$. Thus, $(R, H)$ is aposyndetic at $h$ with respect to $g$ in this special way. Since $H_{B}$ is closed and compact, there exists a finite number of continua $N_{i}, 1 \leqq i \leqq n$, in $(R, H)$ such that $N_{i}^{*}$ is a subcontinuum of $M$. and $\sum N_{i}^{*}$ separates $\ddot{C}$ from $C_{1}$ in $M$. Consequently, $S(g, x) \supset C$. Hence, Theorem 6.2 is true.

The following theorem may be proved by use of previous theorems as well as a well known theorem concerning upper semi-continuous collections of disjoint continua filling up a compact metric continuum.

Theorem 6.3. The hyperspace $(R, G)$ is a compact aposyndetic metric continuum.

Since Theorem 6.2 shows that the elements of $G$ are continua, it is now possible to prove the following theorem with an argument analogous to that given for Theorem 4.4.

THEOREM 6.4. If the collections $H H, H G, G H$, and $G G$ are defined as in $\$ 4$, then $H H=H, H G=H, G H=G$, and $G G=G$.

By defining Property $\mathrm{Y}$ in a special way, a hyperspace which is locally connected may be obtained. Consider the following theorem.

THEOREM 6.5. Suppose that Property Y is the property of being locally connected. Then the hyperspace $(R, G)$ is a compact metric continuous curve.

Proof. By Theorem 6.3, $(R, G)$ is an aposyndetic compact metric continuum. Suppose that $(R, G)$ is not locally connected at some point $a$. Thus, there exist open subsets $D$ and $D_{1}$ of $(R, G)$ such that (1) $D \supset \bar{D}_{1} \supset D_{1} \supset a$ and (2) there exists a collection $C$ of components of $D_{1}$ such that for $k$ in $C$, the component $D_{k}$ of $D$ which contains $k$ has the property that $D_{k} \cdot a=0$; and furthermore, $C$ contains an infinite subcollection $C_{1}$ such that if $C_{2}$ is an infinite subcollection of $C_{1}$, then the closure of $C_{2}$ contains $a$ (see [3]). There exists an infinite subcollection $C_{2}$ of $C_{1}$ such that the limiting set $L$ of $C_{2}$ is a compact continuum in $(R, G)$ containing $a$. For some point $p$ of $M, M(p) \supset a$. Now, there exists a point $g$ of $L$ such that $g$ is not a component of $M(p)$. Hence, there exists an uncountable nonseparated collection $M(p, x, Y)$ of locally connected subsets of $M$ such that each element of $M(p, x, Y)$ separates $M(p)$ from $g$ in $M$.

By use of a theorem due to Whyburn $[9$, p. 45], there exists an uncountable subcollection $M_{1}(p, x, Y)$ of $M(p, x, Y)$ each element of which is closed in $M$. For all elements $k$ in $C_{2}$, let $M\left(C_{2}\right)$ denote the set of all points $y$ in $M$ 
such that $y$ belongs to a point $h$ of $(R, G)$ where $h \in k$. From these definitions of $C_{2}$ and. $L$, it follows that the closure of $M\left(C_{2}\right)$ contains a point from each of the sets $a$ and $g$. Thus, for $e$ in $M_{1}(p, x, Y)$, there exists a subcollection $C_{3}$ of $C_{2}$ such that $C_{2}-C_{3}$ is finite and for $k$ in $C_{3}, e$ separates $k^{*}$ in $M$. Since $k$ is a connected subset of $(R, G)$, it follows that $k^{*}$ is a connected subset of $M$. Now, let $\left\{y_{i}\right\}$ denote a sequence of points such that (1) for each $i$, there exists $k_{i}$ in $C_{3}$ such that $\cdot \cdot k_{i}^{*} \supset y_{i},(2)$ for $i \neq j, k_{i}^{*} \cdot k_{j}^{*}=0$, and (3) $\left\{y_{i}\right\} \rightarrow y \in L^{*}$. Since $e$ is closed, $e \supset y$. By hypothesis, $e$ is locally connected at $y$. Thus, there exists a connected open subset $E$ of $e$ (open relative to $e$ ) containing $y$ such that $E$ is a subset of the component $N$ of $e \cdot D$ which contains $y$. Now, $N \subset L^{*}$. For each $i, N \cdot k_{i}^{*}=0$. Thus, $E$ fails to contain $y_{i}$ for any $i$. This is impossible. Hence, $(R, G)$ is locally connected at each of its points.

The following corollary to Theorem 6.5 is a theorem due to R. L. Moore $[3$, p. 343 (Theorem 23a)].

CoRollary 6.51. Suppose that Property Y is the property of being exactly one point. Then $(R, G)$ is a compact metric acyclic continuous curve.

7. A result for compact plane continua. By use of a characterization of plane continuous curves, i.e., a necessary and sufficient condition that a compact subcontinuum $M$ of the number plane be a continuous curve is that each pair of points in $M$ be separated in $M$ by the sum of a finite number of subcontinua of $M$ [3], the following theorem may be proved.

ThEOREM 7.1. Suppose that $M$ is a compact subcontinuum of the number plane and that Property $\mathrm{Y}$ is the property of being the sum of a finite number or continua. Then $(R, G)$ is a compact metric continuous curve.

The space $(R, G)$ in Theorem 7.1 is not necessarily a subset of the plane. Consider the following.

EXAmple 7.2. Let the points of a space $M$ consist of a bounded spiral in the plane which lies in the exterior of a simple closed curve $J$ on which it spirals down plus $J$ plus the interior of $J$. With the usual topology, $M$ is a compact metric continuum. Now, considering the collection $G$, the set $J$ is the only element of $G$ which is not a point. The hyperspace $(R, G)$ is a sphere plus an arc having only one end point in common with the sphere.

8. Concluding remarks. It is perhaps surprising that Whyburn's definition of collections of nonseparated separators along with the generalization of Moore's sets $M(P)$ [Definitions 2.1 and 2.2] has so many implications even in a separable topological space. Some light seems to have been shed both on these particular collections and sets as well as on the structure of aposyndetic continua. By considering a finite sequence of upper semi-continuous collections filling up $M$ which decompose $M$ into an aposyndetic continuum, knowledge of the structure of $M$ may be obtained. Suppose that $G_{1}, G_{2}, G_{3}, \cdots, G_{n}$ is a finite sequence of upper semi-continuous collections such that $G_{1}$ decom- 
poses $M$ into an aposyndetic continuum while for $1<i \leqq n, G_{i}$ decomposes $G_{i-1}$ into an aposyndetic continuum. With suitable definitions of Property Y, $G_{2}$ may be a continuous curve while $G_{8}$ is an acyclic continuous curve. Of course, a member $G_{i}$ of this sequence may be obtained by use of either the sets $M(p)$ or the sets $S(g, x)$ with one definition of Property Y while $G_{i+1}$ may be obtained by another definition of Property Y. A sequence of this kind constitutes a subspectrum of an aposyndetic spectrum described by F. B. Jones [2].

\section{BIBLIOGRAPHY}

1. F. Burton Jones, Aposyndetic continua and certain boundary problems, Amer. J. Math. vol. 63 (1941) pp. 545-553.

2. Concerning aposyndetic and non-aposyndetic continua, Bull. Amer. Math. Soc. vol. 58 (1952) pp. 137-151.

3. R. L. Moore, Foundations of point set theory, Amer. Math. Soc. Colloquium Publications, vol. 13, New York, American Mathematical Society, 1932.

4. - Fundamental theorems concerning point sets, The Rice Institute Pamphlet vol. 23 (1936) pp. 1-74.

5. J. H. Roberts, Concerning metric collections of continua, Amer. J. Math. vol. 53 (1931) pp. $422-426$.

6. Waclaw Sierpinski, General topology, Trans. by C. Cecilia Krieger, University of Toronto Press, 1952.

7. A. Tychonoff, Über einen Metrisationssatz von P. Urysohn, Math. Ann. vol. 95 (1926) pp. 139-142.

8. Paul Urysohn, Zum Metrisationsproblem, Math. Ann. vol. 94 (1925) pp. 309-315.

9. G. T. Whyburn, Analytic topology, Amer. Math. Soc. Colloquium Publications, vol. 28, New York, American Mathematical Society, 1942.

10. - Concerning collections of cuttings of connected point sets, Bull. Amer. Math. Soc. vol. 35 (1929) pp. 87-104.

11. - Concerning the cut points of continua, Trans. Amer. Math. Soc. vol. 30 (1928) pp. 597-609.

12. - Cut points of connected sets and of continua, Trans. Amer. Math. Soc. vol. 32 (1930) pp. 147-154.

13. - Non-separated cuttings of connected point sets, Trans. Amer. Math. Soc. vol. 33 (1931) pp. 444-454.

14. — Semi-locally-connected sets, Amer. J. Math. vol. 61 (1939) pp. 733-749.

15. C. Zarankiewicz, Über die Zerschneidungspunkte der Zusammenhangende Mengen, Fund. Math. vol. 12 (1928) pp. 121-125.

16. R. G. Lubben, Concerning the decomposition and amalgamation of points, upper semicontinuous collections, and topological extensions, Trans. Amer. Math. Soc. vol. 49 (1941) pp. 410466.

University OF NoRTh Carolina, Chapel Hill, N. C.

UNIVERSITY OF MARYLAND, College Park, MD. 